\title{
Face Detection and Recognition System for Enhancing Security Measures Using Artificial Intelligence System
}

\section{Muhammad Farhan Siddiqui, Waqas Ahmed Siddique, Mushtaq Ahmedh and Awais Khan Jumani*}

\author{
Faculty of Science and Technology, ILMA University, Karachi, Sindh, Pakistan; \\ engrmushtaque@hotmail.com
}

\begin{abstract}
Article Type: Article
Article Citation: Muhammad Farhan Siddiqui, Waqas Ahmed Siddique, Mushtaq Ahmed, Awais Khan Jumani. Face detection and recognition system for enhancing security measures using artificial intelligence system. Indian Journal of Science and Technology. 2020; 13(09),1057-1064.001:10.17485/ijst/2020/ v013i09/149298
\end{abstract}

Received date: November 29, 2019 Accepted date: December 23, 2019

*Author for correspondence: Awais Khan Jumani $\mathbf{V}$ awaisjumani@ yahoo.com $\uparrow$ Faculty of Science and Technology, ILMA University, Karachi, Sindh, Pakistan

\begin{abstract}
Background/objectives: The major changes which come across face recognition are to find the age and gender of the person. This study is centered on face detection with voice and biometric technology. Methodology: During this study, it has been worked on the input camera which takes multiple shots of person. After that, the Cascade Classification algorithm has been used inside the application which creates the multiple human templates. So the facial features have been detected. After that, it saved in particular database with their unique ID. Furthermore, the verification process has been started by matching the templates inside the database. Through this process, the student attendance has been marked automatically. Findings: It has been presented that face detection with voice and biometric technology can enhance the security measures. Employee's attendance can be marked by simply detecting face that can increase punctuality. Students can be checked and marked by face detection process. As well, it can also be setup in banks to enhance the security by allowing authorized people only or who have accounts in that bank. Novelty/improvements: For finding the age and gender from a particular image, relevant techniques are discussed, with some new approaches for maintaining security. We discussed complete models with security measures in this research.
\end{abstract}

Keywords: Face Detection, Image Processing, Distinctive Characteristic Location, Template Creation \& Matching.

\section{Introduction}

This research study involves extracting information like pose, expression, gender, age, identity, etc. which helps to maintain security in an organization [1]. In recent years, face recognition has concerned much attention. This research study will increase human interaction towards technology, as this technology is user-friendly. By using this system, the 
security measures will be enhanced. The main reason for face detection is to find out faces among the peoples and it uses most of the security purposes [2]. Nowadays, face detection is gaining much interest as an important part of the research in many applications, in recent years, face detection has gripped much attention. Face detection is the center part of all facial analysis also it is a fundamental technique for other applications [3].

As the area of artificial intelligence (AI) is still in progress so still, there are some drawbacks. If distortion is found in camera or noise or may be due to changing hairstyles and makeup or may due to face expression or according to the situation. For that we will put an algorithm and few photos of the same person will be stored in the database for better analyzation [4]. The face recognition method in biometrics is one of the most and highly affected security measures. Another more important characteristic of face recognition is passive identification which means this biometric system identifies a person in many different environments. AI is not $100 \%$. It is a vast subject which is still under research, and companies are spending a tremendous amount of money to research and improve this area [5].

In Ref. [6], Kumar et al. have introduced the first Semi-Automated Facial Recognition Technique. In that technique, some of the facial expressions can be recorded and some features have been recorded from the face which are present in the image. The main objective of this research to collect more interest from face recognition and biometric techniques. Many of the companies and educational institutes follow such type of techniques for their betterment of business [7].

The implementation of face recognition consists of four stages [8]

IMAGE ACQUISITION: Facial-scan technology uses to generate images of good quality and resolution by using static camera or video system to acquire faces.

IMAGE PROCESSING: It plays the most important role in face detection and recognition in which unwanted are of image is removed and image is being cropped color image can be converted into black \& white than it shows the grayscale image. First of all, the image should be present into the detected machine than it should be normalized the image.

CHARACTERISTIC LOCATION: In this, we match the visible facial features which include upper ridges of the eye sockets, area around cheekbones, side of the mouth, nose shape these are the major features relative to each other. This feature helps us to identify behavioral changes like hairstyle, makeup, eyeglasses, etc.

TEMPLATE CREATION \& MATCHING: By using multiple processed facial images, we create a template from 100 bytes to over $3 \mathrm{~K}$. In template matching facial detection and recognition system may have 10 to 20 match attempts which take up to 1 to 2 second, as well as Figure 1 shows the pillars of face recognitions [9].

The system works to obtain the value of peaks and valleys of the face and distinguish the landmarks, the visions are a feature which defines these landmarks as nodes points, there are about 80 nodal points on a human face [10]. For the detection of face, there is an algorithm which is used for the face detection named "Viola Jones Algorithm". This algorithm checks the oval shapes in our face, which includes our eyes, nose, mouth, etc. which helps to recognize the face properly [11]. The one of the main advantages of face detection and recognition is if you have a powerful camera system then you can easily 


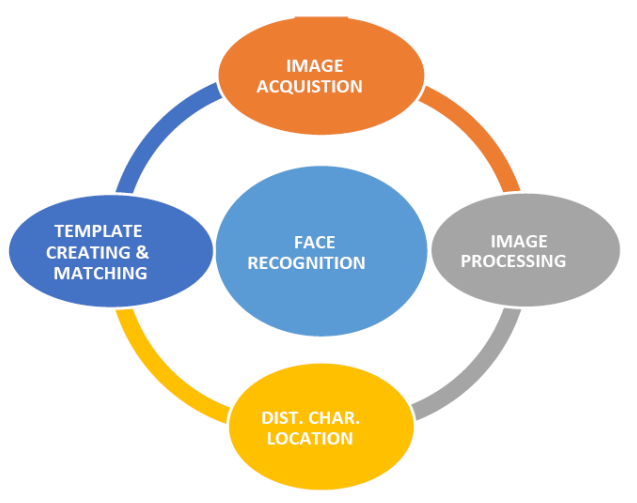

FIGURE. 1. Face recognitions pillars.

target the specific area in which some peoples were present, which will enable us to identify does any criminal is present between them or not, who is standing far from you. We can use this technology system in public places, airport, and stadium, etc [12].

\section{Problem Identification}

The problem statement regarding this research is security issues related to "our security systems" that needs to be more improved to reduce our security risks are in daily life, for this purpose, we have addressed these issues in our research paper and suggested the solution as face detection and recognition system for enhancing security measures using AI. Face recognition is the biometric method currently enhanced by human beings to improve security systems, but it has also a greater risk. There are categorized into two factors.

- INTRINSIC FACTOR: age, facial hair, glasses, cosmetics gender, etc.

- EXTRINSIC FACTOR: illumination, focus, imaging, resolution, pose, etc. [13]

In face detection technique, sometimes the camera could not recognize the person even their data are saved in the database. Few pictures of the same person will be saved in data. If case of hair color change, age increase and dress color, and the system will be analyzed easily [14]. Similarly, most of the research related to feature selection focuses on face recognition and their classifications. Regarding 3D pictures, most of the thing is impossible to take but it needs more important to take good performance in it [15]. In illumination, the problem face appears different due to light changing so the camera could not find out the enough information. In pose problems face appears different due to changing in view condition. Basically, as all we know technology is increasing day by day and on the other hands our security systems need to be improved and more secure, the problem is that if we do not upgrade or change our security systems then it will become as big risk for security, from this stage our research purpose has started and we have chosen the face detection and recognition technology for enhancing our security measures [16]. The author has worked on video recording of chimpanzee through deep learning 
of neural network. In this work, he has classified the chimpanzee using facial detection after that it analyzes the behavior from long-term video recording [17]. Similarly, another has classified the text using support vector machine in this system he has classified those which are required [18]. Similar work on the same approach for the classification of text was used in natural language processing [19-20].

The usage of AI enables that no one can have unauthorized access or become risky for our security systems. We have specially selected this topic because this technology is very effective and also this can be implemented in various platforms and areas like school/ college/university, airport, stadium, parks, and other public areas. If we have a powerful camera system, we would be able to detect any person's face by using face detection and recognition system. As it will help us to identify the person's identity, we can easily know about their profile. For the development of this software, we are using python language with Open CV which is very compatible for the development of this software. This technology generally targets the specific person and as well as many persons in public areas to identify their identity.

\section{Methodology}

An input camera device is required to take the multiple shots of the object/person. As for the algorithm, Cascade classification is used for creating the multiple templates of the facial and detects facial features. A database is used for storing the templates along with student's roll number which acts as unique ID. Throughout the verification process, the camera detects the facial features and tries to match against the templates which are already stored in the database; if found then it runs through the attendance management system process and marks the attendance for a particular student otherwise absent will be marked for not present students.

\subsection{Components}

In order to use this system, it requires we have some requirements

\subsubsection{Hardware Requirements}

- Personal Computers

- An Input Camera/Webcam

- Active Internet Connection

\subsubsection{Software Requirements}

- Python development environment and Open CV which is written in $\mathrm{C} / \mathrm{C}++$ which uses a powerful machine learning algorithm for search facial features within a picture. It runs through thousands of facial patterns and matching the facial features.

- Postgre SQL is used for creating the database of the system.

- Tkinter is used for creating the whole interface of the software. 


\subsection{GSM SMS Notification}

SMS notifications will be sent to the enrolled students. This can also work for students who has the below $70 \%$ of attendance and also for those who has not paid their fees. So whenever the student passes by the camera so their attendance and fees will be checked on the fly. In the case, Figure 2 shows the administration login. In this Figure 3 shows the direct face capturing where the student's picture has been shown. In this Figure 4 use case diagrams has been shown it shows the connectivity of admin and students.

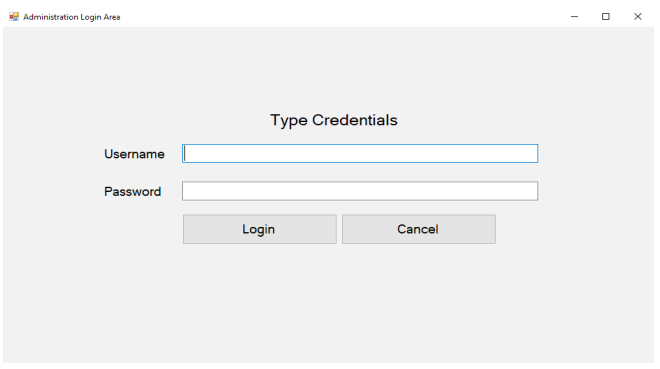

FIGURE. 2. Illustrates the administration login.

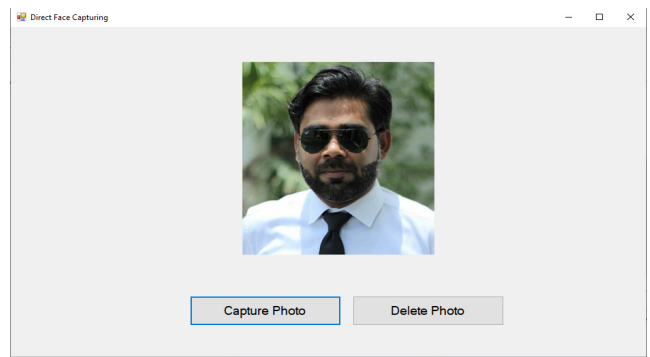

FIGURE. 3. Direct face capturing.

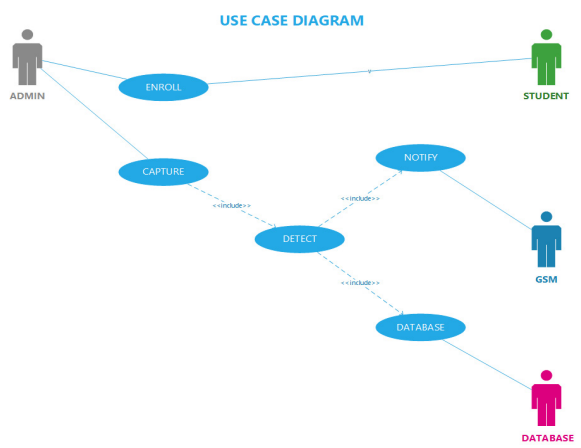

FIGURE. 4. Use case diagram of face detection.

\subsection{Use Case Diagram}

Figure 3 shows the interaction between the user and the software which shows how the actual action is being performed and interacts with the user hence it is considered as the blueprint diagram of the system. 


\subsection{Database}

The database holds the user information such as title, middle name, surname, date of birth, gender, nationality, etc. Each record has storage of images minimum ten images and maximum fifty images of each student record. See Figures 4 and 5 for the interface of enrolling. Verification is carried out through this student identification portal if the template is matched against the user then the present is marked for the day.

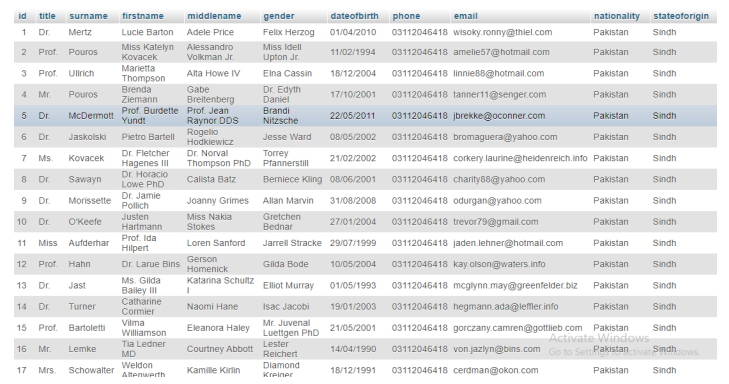

FIGURE 5. Database structure.

In Figure 6, it shows the student bio data. In this manner, all information regarding student can be collected.

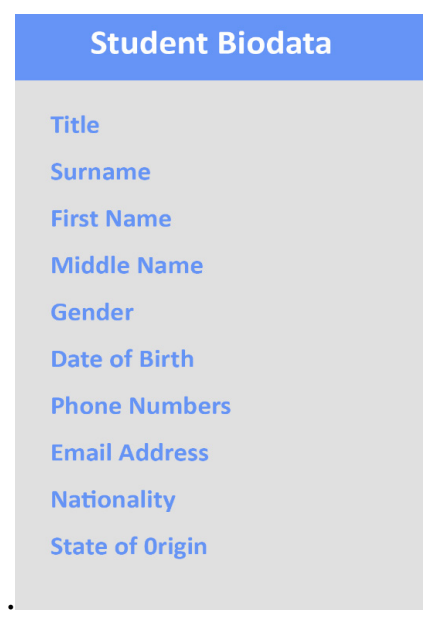

FIGURE. 6. Bio-data form.

\section{Discussion \& Conclusion}

To check the accuracy of the system, some tests are done as given below:

- Various facial expressions

- Various lighting conditions

- These tests are performed on ten individual records in the system. 
The system responded better to various facial expressions, but sometimes got hard time to recognize the faces on the lighting conditions. Thus, the facial recognition and attendance management system can be used for only for good lighting conditions otherwise it will get problems to recognize the faces as light serves as the primary eye of the camera; however, system performed quite well with the facial expressions. Creative visualization is a great way to see a possible future and move you towards it. Smartphone technologies give us lot of facilities in better education. Many of the students take interest to use smartphone in classrooms and the majority of the students want to learn to visualize technologybased learning. So many of the software and tools have been introduced for learning with technology but still they do not fulfill students' requirements. We have proposed an application which fulfils our student's needs as compared to traditional learning system. The experiment has been based on ASP.NET and CSS framework, and also it has an ability to retrieve previous records from database.

\section{Future Work}

Embed Systems such as Arduino, Raspberry Pi, and others can be incorporated easily and take the benefits of this system not only as the attendance system but also as the security system too and it can be developed in various other industries as AI has become a need of every company.

\section{References}

1. Naeem M, Qureshi I, Azam F. Face recognition techniques and approaches: a survey. Science International. 2005, 27 (1), 1-5.

2. Parkhi OM, Vedaldi A, Zisserman A. Deep face recognition. In: BMVC. 2015; vol 3, 1-12.

3. Chauhan M, Sakle M. Study \& analysis of different face detection techniques. International Journal of Computer Science and Information Technologies. 2014, 5(2), 1615-1618.

4. Hassaballah M, Aly S. Face recognition: challenges, achievements and future directions. IET Computer Vision. 2015, 9(4), 614-626.

5. Bhatia R. Biometrics and face recognition techniques. International Journal of Advanced Research in Computer Science and Software Engineering. 2013, 3(5), 1-7.

6. Kumar S, Singh S, Kumar J. A study on face recognition techniques with age and gender classification. In: 2017 International conference on computing, communication and automation. 2017; 1001-1006.

7. Turk MA, Pentland AP. Face recognition using eigenfaces. Proceedings. 1991 IEEE Computer Society conference on computer vision and pattern recognition. 1991, 586-591.

8. Bowyer KW. Face recognition technology: security versus privacy. IEEE Technology and Society Magazine. 2004, 23(1), 9-19.

9. Gürel C, Erden A. Design of a face recognition system. Proc. the 15th Int. conference on machine design and production. 2012.

10. Wiskott L, Fellous JM, Krüger N, Von Der Malsburg C. Face recognition by elastic bunch graph matching. In: International conference on computer analysis of images and patterns. Springer: Berlin, Heidelberg. 1997; 456-463. 
11. Hossen AMA, Ogla RAA, Ali MM. Face detection by using open CV's Viola-Jones algorithm based on coding eyes. Iraqi Journal of Science. 2017, 58(2A), 735-745.

12. Hsu RL, Abdel-Mottaleb M, Jain AK. Face detection in color images. IEEE Transactions on Pattern Analysis and Machine Intelligence. 2002, 24(5), 696-706.

13. Bowyer KW, Chang K, Flynn P. A survey of approaches and challenges in 3D and multi-modal 3D+2D face recognition. Computer Vision and Image Understanding. 2006, 101(1), 1-15.

14. Hatem H, Beiji Z, Majeed R. A survey of feature base methods for human face detection. International Journal of Control and Automation. 2015, 8(5), 61-78.

15. Xiaoqing D, Chi F. Discussion on some problems in face recognition. Proceedings of the international conference on advance in biometric person authentication, Guangzhou, China. 2004, 47-56.

16. Cascio WF, Montealegre R. How technology is changing work and organizations. Annual Review of Organizational Psychology and Organizational Behavior. 2016, 3, 349-375.

17. Schofield D, Nagrani A, Zisserman A, Hayashi M, Matsuzawa T, Biro D, Carvalho S. Chimpanzee face recognition from videos in the wild using deep learning. Science Advances. 2019 Sep 1, 5(9), eaaw0736.

18. Shujrah AA, Rajper S, Jumani AK. Measurement of e-learners' level of interest in online course using support vector machine. Indian Journal of Science and Technology. 2019 Oct, 12, 40.

19. Jumani AK, Memon MA, Khoso FH, Sanjrani AA, Soomro S. Named entity recognition system for sindhi language. In: International conference for emerging technologies in computing. Springer: Cham. 2018 Aug 23; 237-246.

20. Jumani A, Mahar M, Khoso F, Memon M. Online text categorization system using support vector machine. Sindh University Research Journal-SURJ (Science Series). 2018 Mar 7, 50(01), $85-90$. 